LIMA, MJA; FARIAS, VDS; COSTA, DLP; SAMPAIO, LS; SOUZA, PJOP. 2016. Efeito combinado das variáveis meteorológicas sobre a condutância estomática do feijão-caupi. Horticultura Brasileira 34: 547-553. DOI - http://dx.doi.org/10.1590/S0102-053620160414

\title{
Efeito combinado das variáveis meteorológicas sobre a condutância estomática do feijão-caupi
}

\author{
Marcus JA Lima'; Vivian DS Fariasi; Deborah LP Costa ${ }^{2}$; Leila S Sampaio'; Paulo JOP Souza' \\ ${ }^{1}$ Universidade Federal Rural da Amazônia (UFRA), Belém-PA, Brasil; marcuslima01@yahoo.com.br; viviandielly19@yahoo.com.br; \\ paulo.jorge@ufra.edu.br; leila.sampaio@ufra.edu.br; ${ }^{2}$ Instituto Tecnológico Vale (ITV), Belém-PA, Brasil; debobahpires.agro@gmail. \\ com
}

\section{RESUMO}

A condutância estomática ( $g s)$ é uma variável fisiológica que faz referência ao fluxo gasoso $\left(\mathrm{CO}_{2}\right.$ e $\left.\mathrm{H}_{2} \mathrm{O}\right)$ entre folha e atmosfera, portanto, representa um processo fundamental para as pesquisas agrometeorológicas. O objetivo deste estudo foi ajustar e validar um modelo descritivo, para quantificar o efeito das variáveis meteorológicas que modulam a gs como: temperatura do ar (T), irradiância solar $\left(\mathrm{I}_{\mathrm{o}}\right)$, déficit de pressão de vapor no ar (DPV) e umidade no solo. O modelo, proposto neste estudo foi calibrado com dados experimentais realizados em blocos casualizados. A gs potencial ( $g s p$ ) (condições ótimas de água no solo) foi estimada a partir do produto das variáveis meteorológicas $\left(\mathrm{T} \times \mathrm{DPV} \times \mathrm{I}_{0}^{-1}\right)$. Na sequência a gsp foi corrigida para a condição atual de água no solo, utilizando um fator de estresse hídrico. As estimativas de $g s$ obtidas pelo modelo foram comparadas com dados experimentais independentes. Os testes de validação indicaram que o modelo foi satisfatoriamente sensível às variações meteorológicas e aos teores de água disponíveis no solo, a julgar pelo baixo erro absoluto médio das estimativas $(<15 \%)$ e pela alta concordância entre os dados observados e simulados $(\mathrm{d}=0,82)$.

Palavras-chave: Vigna unguiculata, modelo dinâmico de cultura, evapotranspiração.

\begin{abstract}
Joint effect of meteorological variables on cowpea stomatal conductance

Stomatal conductance $(g s)$ is a physiological variable related to gas exchange $\left(\mathrm{CO}_{2}\right.$ and $\left.\mathrm{H}_{2} \mathrm{O}\right)$ between leaf and atmosphere. Therefore, $g s$ is a fundamental process for meteorological research. The aim of this study was to adjust and validate a descriptive model to quantify the effect of the main environmental variables that modulate $g_{s}$ such as air temperature $(T)$, solar irradiance $\left(\mathrm{I}_{\mathrm{o}}\right)$, vapor pressure deficit in the air (VPD) and soil moisture. The model proposed in this study was calibrated with experimental data carried out in a randomized block design. The potential gs (optimum conditions of soil water) was estimated as a function of the meteorological variables product $\left(\mathrm{T} \times \mathrm{DPV} \times \mathrm{I}_{0}^{-1}\right)$. Following, the potential $g s$ was adjusted to the current condition of soil water, using a water stress factor. The gs values estimated by the model were compared with independent experimental data. Validating tests indicated that the model was satisfactorily sensitive to weather variations and water content available in the soil, due to a low mean absolute error of the estimates $(<15 \%)$ and a high agreement between the observed and simulated data $(\mathrm{d}=0.82)$.
\end{abstract}

Keywords: Vigna unguiculata, crop dynamic model, evapotranspiration.

(Recebido para publicação em 22 de outubro de 2015; aceito em 1 de abril de 2016) (Receivid on October 22, 2015; accepted on April 1, 2016)

$\mathrm{O}$ feijão-caupi (Vigna unguiculata) se destaca como uma das culturas mais cultivadas na região nordeste do estado do Pará. A atividade do cultivo desta cultura é considerada a principal fonte de emprego e renda nessa região, onde cerca de $90 \%$ da produção tem sido comercializada para estados nordestinos. O nordeste paraense vem se notabilizando pela forte incorporação de tecnologia ao sistema produtivo, almejando reduzir riscos e planificar a produção (Rebello et al., 2011). Neste contexto, a adoção de modelos agrometeorológicos capazes de simular o rendimento da cultura, torna-se uma excelente ferramenta de planejamento.

Os modelos agrometeorológicos também chamados de modelos dinâmicos de cultura (MDC) têm sido amplamente utilizados como ferramentas de suporte à tomada de decisão na pesquisa agronômica, produção agrícola e planejamento de uso da terra. Esses modelos são frequentemente utilizados em estudos de riscos climáticos atuais e futuros
(Moradi et al., 2013) e de otimização de rendimento por meio de estratégias de manejo como densidade de semeadura, adubação e irrigação (Barterng et al., 2010). Por exemplo, Aristides et al. (2013), utilizando um MDC, determinaram, satisfatoriamente, a melhor época de semeadura do feijão-caupi pelo agrupamento das datas de semeadura que apresentaram os maiores valores e menor variabilidade de rendimento simulado na região do Recôncavo Baiano.

Os MDC estão estruturados em 
submodelos, que simulam processos ordenados do crescimento vegetal. A grande maioria deles é considerada semimecanística, ou seja, apenas uma parte de sua estrutura, baseia-se em teoria fisiológica sendo necessário um modelo empírico (baseado em estatística) para complementar o processo. Esse fato faz com que os MDCs apresentem características da região para a qual foram desenvolvidos, necessitando portanto, de algum tipo de modificação para uma correta utilização fora de seu lugar de origem (Souza et al., 2011).

A estimativa da evapotranspiração representa um dos principais submodelos dos MDC por estar intimamente relacionado com o sequestro de carbono e com o rendimento das culturas; além disso, a sua estimativa é fundamental para um correto manejo da água. A equação de Penman-Monteith quando associada à variável de condutância estomática $(g s)$ pode estimar diretamente a evapotranspiração da cultura (ETc) para distintas condições atmosféricas e, ou, umidade do solo (Ortega-Farias et al., 2004), necessitando para isso, de uma estimativa precisa de $g s$ para a cultura em questão.

A gs é uma variável fisiológica que controla o fluxo de água do mesófilo foliar para atmosfera, sendo sensível a múltiplos fatores ambientais. Dada sua importância, diversos modelos foram desenvolvidos para explicar a influência não linear dos fatores ambientais na variabilidade da $g s$ (Damour et al., 2010). Dentre eles, os mais importantes pela ampla utilização são os modelos de Jarvis (1976) e de Ball et al. (1987). Entretanto, a principal limitação do modelo de Jarvis (1976) tem sido a falta de interatividade entre as variáveis ambientais, enquanto que o modelo de Ball et al. (1987) necessita ser acoplado a um complexo modelo de fotossíntese, nem sempre disponível para cultura de interesse. Ressalta-se ainda que, nenhum desses modelos incluiu diretamente o efeito do déficit hídrico na estimativa de gs (Damour et al., 2010).

Apesar das numerosas publicações sobre o mecanismo de resposta dos estômatos aos estímulos ambientais, ainda não há um modelo amplamente aceito, pois a $g s$ pode variar em função de diversos fatores climáticos, genéticos e sistema de manejo, tornando os modelos, simplificados, de difícil validação (Lobet et al., 2014).

A estimativa da gs por meio de um modelo calibrado regionalmente para a Amazônia poderá melhorar as simulações dos MDCs nesta região, quanto as estimativas de acumulo de biomassa, rendimento e consumo hídrico (Souza et al., 2011), desta forma auxiliando nos estudos de mitigação dos impactos de eventos climáticos extremos na produção agrícola, como El Niño e La Niña, os quais poderão ocorrer com maior frequência no futuro devido aos efeitos das mudanças climáticas (Tack \& Ubilava, 2015).

Diante das premissas apresentadas, o objetivo de estudo foi ajustar e validar um modelo empírico para quantificar a relação entre variáveis meteorológicas (temperatura do ar, déficit de pressão de vapor do ar e irradiância solar), associadas ao efeito do déficit hídrico na variabilidade da condutância estomática para cultura do feijão-caupi.

\section{MATERIAL E MÉTODOS}

Os experimentos foram realizados na Fazenda Escola de Castanhal-PA (FEC-UFRA), localizada nas proximidades da BR 316 (1 ${ }^{\circ} 19^{\prime} 24^{\prime \prime} \mathrm{S}$, 47'57'38'O, $41 \mathrm{~m}$ de altitude) no município de Castanhal, nordeste do Estado do Pará, Norte do Brasil, durante os anos de 2012-2014. O solo da área foi classificado como Latossolo Amarelo distrófico com horizonte B latossólico. O clima da região foi classificado como Ami, segundo a classificação de Köppen, com temperatura média anual de $26^{\circ} \mathrm{C}$ e as temperaturas máxima e mínima variando entre $35^{\circ} \mathrm{C}$ e $20^{\circ} \mathrm{C}$, respectivamente. A umidade relativa do ar em média foi de $80 \%$ e precipitação média anual de 2.000 a $2.500 \mathrm{~mm}$.

O delineamento experimental foi de blocos casualizados, com seis repetições. Cada bloco foi dividido em quatro unidades experimentais de 100 $\mathrm{m}^{2}(5 \times 20 \mathrm{~m})$ espaçadas de dois metros. Os tratamentos consistiram de quatro lâminas de irrigação [100, 75, 50 e $25 \%$ da evapotranspiração da cultura (ETc)].
A redução das lâminas iniciou a partir do início do florescimento e permaneceram até a maturação fisiológica.

Foi utilizada a cultivar BR3-Tracuateua, de hábito de crescimento indeterminado, porte prostrado, ciclo de 60-70 dias, muito utilizada no nordeste paraense. As semeaduras foram realizadas em 26 de setembro de 2012; 2 de outubro de 2013 e 9 de setembro de 2014 de forma mecanizada, de modo a obter aproximadamente 10 plantas por metro linear com espaçamento entre linhas de $50 \mathrm{~cm}$, (200.000 plantas/ha). A adubação de fundação consistiu na aplicação de 15-60-60 kg/ha de NPK. Os experimentos foram conduzidos, de modo a manter a área livre de plantas invasoras, doenças e pragas. Aos 25 dias após a semeadura (DAS) aplicou-se macro e micronutrientes via foliar $(30 \mathrm{~kg} / \mathrm{ha}$ de FTE BR 12), visando a padronização do desenvolvimento da cultura.

Foi adotado o sistema de irrigação por gotejamento, utilizou-se uma mangueira de $16 \mathrm{~mm}$ com gotejador tipo labirinto espaçados de $20 \mathrm{~cm}$ com vazão de $0,94 \mathrm{~L} / \mathrm{h}$ sob pressão de $10 \mathrm{mca}$, para cada linha de plantio. O manejo da irrigação foi baseado na evapotranspiração de referência estimada por Penman-Monteith e os coeficientes de cultura (Kc) obtidos por Bastos et al. (2008). Os dados meteorológicos (temperatura e umidade relativa do ar, radiação solar, velocidade do vento) utilizados foram baixados diariamente do site do Instituto Nacional de Meteorologia (INMET), referente a uma estação meteorológica automática situada aproximadamente 2 $\mathrm{km}$ da área experimental. As lâminas de água aplicadas em cada parcela foram controladas em função do tempo, de acordo com a vazão dos emissores, utilizando-se manômetros, registros e cronômetro. A cada dois dias determinava-se o tempo de irrigação para a parcela de referência ( $100 \%$ da ETc), e, posteriormente, as lâminas com reduções de 75,50 e $25 \%$ da ETc, respectivamente (Carvalho et al., 2014).

A $g s$ foi medida com uso do porômetro (AP4/Delta-TDevices) em dias pré-definidos nos estádios fenológicos: fim da fase vegetativa (V4), floração (R6), enchimento de grão (R8) e maturação fisiológica (R9). As medidas foram 
realizadas ao longo do dia, das 8 às 16 horas com intervalo de uma hora. Desta forma, quatro plantas foram tomadas ao acaso, e a $g s$ foi medida na face abaxial do terceiro trifólio da haste principal completamente expandida (Paiva et al., 2005).

$\mathrm{Na}$ área experimental foi instalada uma estação automática, equipada com radiômetro, psicrômetro e pluviômetro. Em cada tratamento (100, 75, 50 e 25\% da ETc) utilizou-se um sensor TDR (reflectometria no domínio do tempo) para a medição da umidade do solo. Os sensores e os equipamentos TDR foram conectados a um datalogger (CR1000 da Campbell), configurado para armazenar as médias a cada 10 minutos. $O$ déficit de pressão de vapor (DPV) foi calculado a partir dos dados de temperatura e umidade relativa do ar, expresso em $\mathrm{kPa}$ (Rodrigues et al., 2011). A umidade do solo, medida com o uso de TDR, foi convertida em fração de água disponível do solo (F) de acordo com a Equação 1 (Lima et al., 2006):

$$
F=\frac{\theta_{i}-\theta_{p m p}}{\theta_{c c}-\theta_{p m p}}
$$

em que F é a fração de água disponível remanescente no solo; $\theta_{i}$ é a umidade atual de água no solo medida pelo TDR, $\mathrm{m}^{3} / \mathrm{m}^{3} ; \theta_{\text {cc }}$ e $\theta_{\text {pmp }}$ representam a umidade do solo na capacidade de campo e no ponto de murcha permanente, expressas em $\mathrm{m}^{3} / \mathrm{m}^{3}$, cujos valores utilizados foram 0,21 e 0,09, respectivamente, os quais foram previamente determinados por gravimetria na camada de $0-30 \mathrm{~cm}$.

A hipótese deste estudo presume que a gs atual ( $g s a)$, pode ser obtida a partir da $g s$ potencial $(g s p)$, medida realizada em campo sob ótimas condições hídricas, no período entre 11 e 13 horas em dias de céu limpo, no estádio fenológico (R6) mais ativo em relação a gs (Paiva et al., 2005). Posteriormente a gsp pode ser ajustada para o real teor de água no solo, por meio de uma função de penalização (Fp), Equação 2:

$$
g f_{a}=g f_{p} \times F p
$$

O modelo foi calibrado utilizando $50 \%$ dos dados do experimento realizado em 2014, onde os valores de $g s$ obtidos no tratamento $100 \%$ da ETc foram ajustados a uma curva gaussiana, em função do produto das variáveis meteorológicas (Equação 3) para estimar a $g s p$ :

$$
\begin{gathered}
g s_{p}=g s_{\max } \times e^{\left(-e^{(-z)}-z+1\right)}-g s_{i m} \\
z=\frac{\text { prod }- \text { fin }}{\text { ini }}
\end{gathered}
$$

$$
\text { prod }=T \times D P V \times I o^{-1}
$$

em que $g s_{\max }, g s_{i m}$, ini e fin representam respectivamente a gs máxima medida em campo e o incremento mínimo, enquanto que ini e fin indicam o intervalo em que o prod contribui para máxima $g s, \mathrm{~T}$, DPV e I correspondem a valores médios de temperatura média do $\operatorname{ar}\left({ }^{\circ} \mathrm{C}\right)$, déficit de pressão de vapor no $\operatorname{ar}(\mathrm{kPa})$ e irradiância solar global $(\mathrm{kJ} / \mathrm{h})$ obtidos entre 11 e 13 horas e $\mathrm{z}$ representa um fator de ponderação em função do produto das variáveis meteorológicas (prod).

O fator de penalização (Fp) expressa a redução relativa da $g s p$ em função da fração de água atual disponível no solo (F). O valor de Fp pode variar de 0 a 1 sendo 1 para condições ótimas de água no solo e 0 para condição de ponto de murcha permanente. A Equação 6 tem sido utilizada, da mesma forma, no MDC de soja de Sinclair (1987), para penalizar a expansão foliar e absorção de nitrogênio. Neste estudo, o ajuste se deu pela regressão dos dados de $g s$ normalizados pela $g s_{\text {max }}$ contra os valores de F dos respectivos tratamentos de água no solo do experimento de 2014.

$$
F p=\frac{A}{1+e^{(-b \times(F-c))}}-1
$$

em que $A, b$ e $c$ são parâmetros de ajuste. $\mathrm{O}$ valor de $\mathrm{F}$ pode variar de $0 \mathrm{a}$ 1 , quando $\theta \mathrm{i}$ varia entre o $\theta_{\mathrm{pmp}}$ e a $\theta_{\mathrm{cc}}$, respectivamente; desta forma o valor de $\mathrm{F}$ é reduzido diariamente devido a evapotranspiração, mas pode aumentar pela adição de água via irrigação e/ou precipitação.

O modelo estima a gs em escala diária em função de dados médios de T, DPV e I obtidos entre 11 e 13 horas, pois neste período os valores de gsp apresentaram menor dispersão.
As estimativas de $g s$ foram comparadas com os valores obtidos experimentalmente em campo nos anos de 2012, 2013 e 2014, com exceção daqueles utilizados na calibração, 50\% dos dados de 2014. Os testes de avaliação empregados foram erro absoluto médio (EAM) (Equação 7), o índice $d$ de Willmott, (Equação 8), eficiência do modelo (Ef) (Equação 9) e como informação complementar o coeficiente de determinação $\left(\mathrm{R}^{2}\right)$.

$$
\begin{gathered}
E A M=\frac{\sum_{i=1}^{n}\left|y_{i}-\hat{y}_{i}\right|}{n} \quad(7) \\
d=1-\frac{\sum_{i=1}^{n}\left(y_{i}-\hat{y}_{i}\right)^{2}}{\sum_{i=1}^{n}\left(\left|\hat{y}_{i}-\bar{y}_{i}\right|+\left|y_{i}-\bar{y}_{i}\right|\right)^{2}} \\
E f=1-\frac{\sum_{i=1}^{n}\left(y_{i}-\hat{y}_{i}\right)^{2}}{\sum_{i=1}^{n}\left(y_{i}-\bar{y}_{i}\right)^{2}} \text { (9) }
\end{gathered}
$$

em que, $y, \hat{y}$ e $\bar{y}$ representam respectivamente a $g s$ observada, estimada, e a média da observada, ambos em $\mathrm{mmol} / \mathrm{m}^{2} / \mathrm{s}$, e $n$ é o número de observações.

\section{RESULTADOS E DISCUSSÃO}

Durante o ciclo da cultura, as temperaturas máxima e mínima foram de $20^{\circ} \mathrm{C}$ e $35^{\circ} \mathrm{C}$, o DPV e irradiância solar máximos foram de $2,5 \mathrm{kPa}$ e $2500 \mathrm{~kJ}$, respectivamente, com pouca variabilidade diurna e entre os anos experimentais (Figuras 1A, 1B e 1C). Tais resultados encontram-se dentro dos padrões normais da região, pois, a maior parte do estado do Pará apresenta pequenas variações de temperatura, umidade, irradiância e vento, devido, em grande parte, sua posição geográfica, porém, pelo mesmo motivo, apresenta elevados índices pluviométricos, com duas estações bem definidas, uma chuvosa outra menos chuvosa.

Na Figura 1D encontra-se o padrão de variabilidade diurna da $g s$, onde observa-se certa semelhança com o padrão da T, DPV e I , evidenciando uma correlação da gs com as variáveis ambientais, supracitadas. Monteith (1995) 
analisou a resposta da $g s$ de diversas espécies (não incluindo o feijão-caupi) em câmara fechada, e identificou três regimes de resposta para DPV: (i) um aumento aproximadamente linear em baixos valores de DPV, (ii) seguido de uma constância ou início de redução, devido ao gradual fechamento estomático e (iii) decréscimo em elevados valores de DPV.

Para as condições de campo deste trabalho, foi observado um padrão semelhante ao descrito por Monteith (1995). Uma análise mais detalhada, em escala horária, do efeito do DPV e da I permitiu um maior entendimento dos regimes supracitados. Por exemplo, durante a primeira metade do dia (6-12h) houve aumento proporcional tanto do DPV quanto da I, indicando que nesse momento ambas variáveis induzem o aumento da $g s$, porém logo após as $12 \mathrm{~h}$ a gs respondeu quase que imediatamente à redução da intensidade luminosa, dando início ao segundo regime e um efeito contrário da DPV, pois esta variável continua aumentando até as $14 \mathrm{~h}$ (Figura 1D).

A rápida resposta da $g s$ à radiação solar também foi observada por Pieruschka et al. (2010) em experimentos com câmara fechada sob vários níveis de irradiância solar. De acordo com Gao et al. (2002), o aumento da intensidade luminosa induz o aumento da concentração de potássio na célula guarda, causando redução do potencial osmótico e consequente aumento na turgescência, portanto, induzindo a abertura estomática. Experimentos em campo com a cultura do feijão-caupi corroboram tais resultados, ao evidenciarem a redução da $g s$ logo após a irradiância máxima do dia (Silva et al., 2010). Todavia, o efeito do DPV é amplamente aceito na literatura especializada e seu aumento tem efeito direto na transpiração e consequente redução da $g s$. De acordo com Vico et al. (2013), isto se deve ao aumento da transpiração cuticular, diminuindo assim o fluxo de água das células epidermicas, principais fornecedoras de água para as células guardas, impactando negativamente na turgescência das mesmas, porém em condições de campo a redução da $g s$ parece estar mais relacionada com a

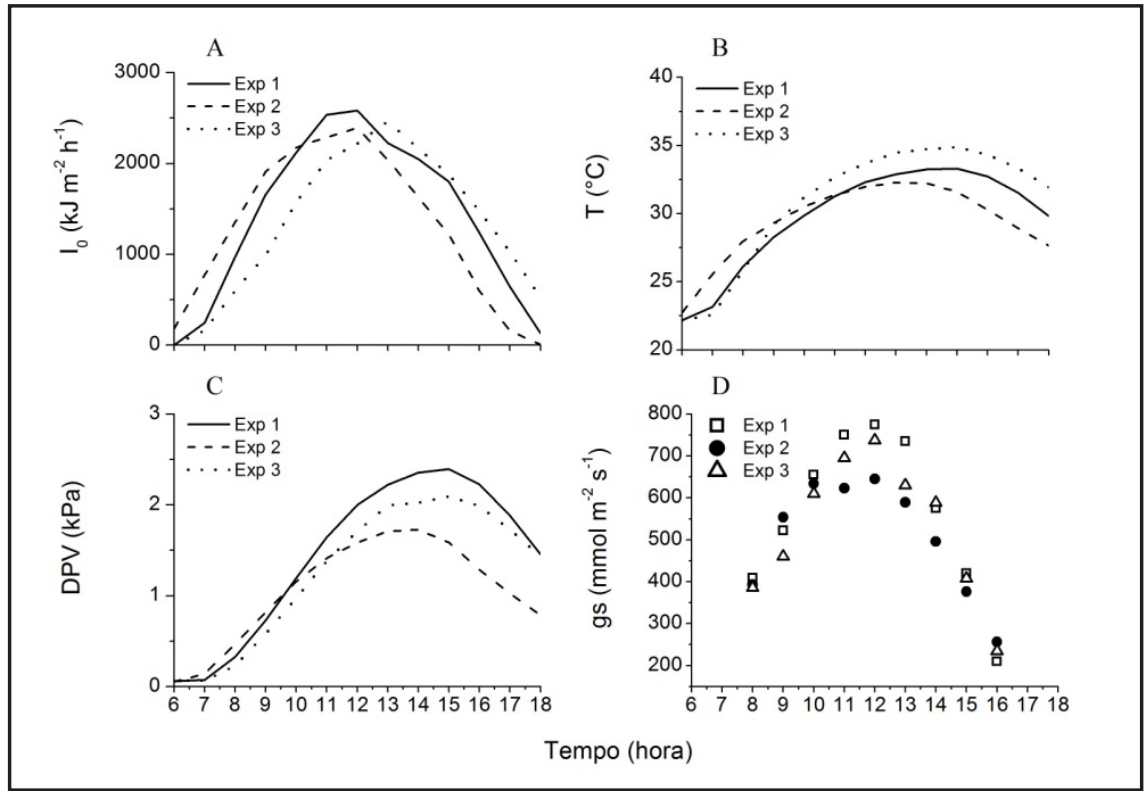

Figura 1. Variabilidade diurna da irradiância solar $\left(I_{0}\right)(A)$, temperatura média do ar (T) (B), déficit de pressão de vapor do ar (DPV) (C) e condutância estomática ( $g s)(\mathrm{D})$ durante o estádio R6, em dias de céu limpo. Dados obtidos nos três anos experimentais: 2012, 2013 e 2014 (exp. 1, 2 e 3, respectivamente) \{diurnal variability of solar radiation $\left(\mathrm{I}_{\mathrm{o}}\right)(\mathrm{A})$, average air temperature $(\mathrm{T})(\mathrm{B})$, vapor pressure deficit in the air $(\mathrm{VPD})(\mathrm{C})$ and stomatal conductance ( $g s)(\mathrm{D})$ during the R6 stage, in clear sky days. Experimental data obtained in three years 2012, 2013 and 2014 (Exp.1, 2 and 3, respectively)\}. Belem, UFRA, 2012-2014.

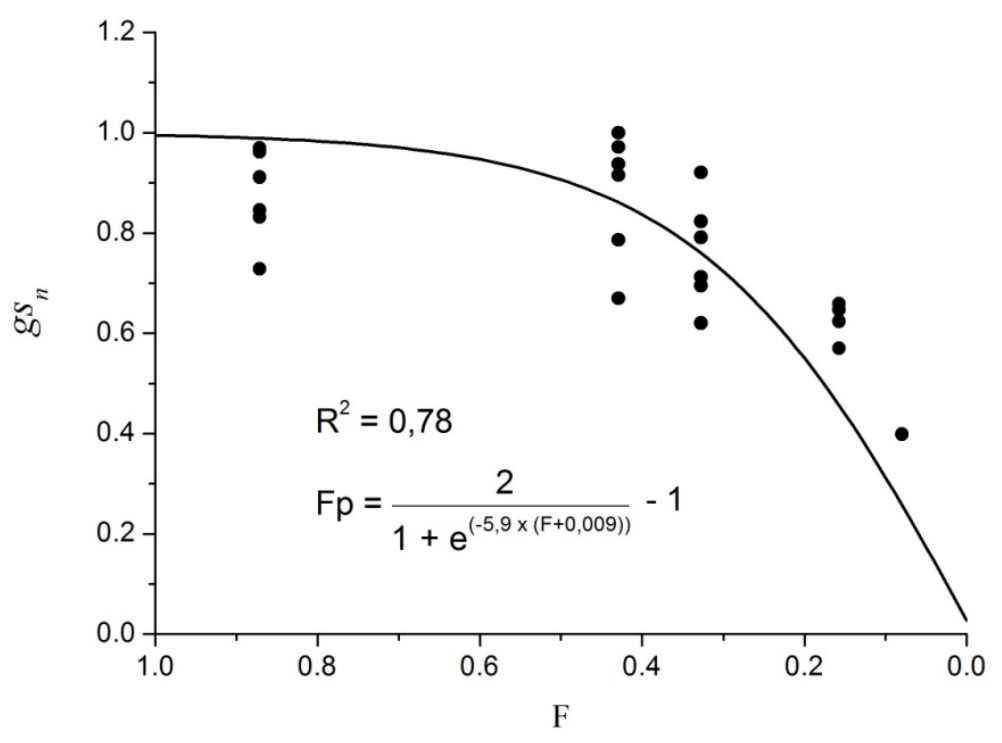

Figura 2. Relação entre fração de água disponível do solo (F) e condutância estomática normalizada $\left(g s_{n}=g s / g s_{\max }\right)$ \{relation between available water fraction in the soil $(\mathrm{F})$ and standardized stomatal conductance $\left.\left(g s_{n}=g s / g s_{\max }\right)\right\}$. Belem, UFRA, 2012-2014.

redução da $I_{0}$.

O monitoramento da umidade do solo nos tratamentos T1, T2, T3 e T4 indicou valores médios correspondentes a $87,43,33$ e $16 \%$ demonstrando que houve um gradiente de estresse hídrico na fase reprodutiva. No entanto, em média, não foram verificadas grandes diferenças de $g s(<20 \%)$ para uma disponibilidade hídrica de até $35 \%(\mathrm{~F}=$ 0,35 ) (Figura 2), o qual pode ser explicado, em parte, pelo eficiente sistema osmorregulador da cultura.

Experimentos realizados em casa de 


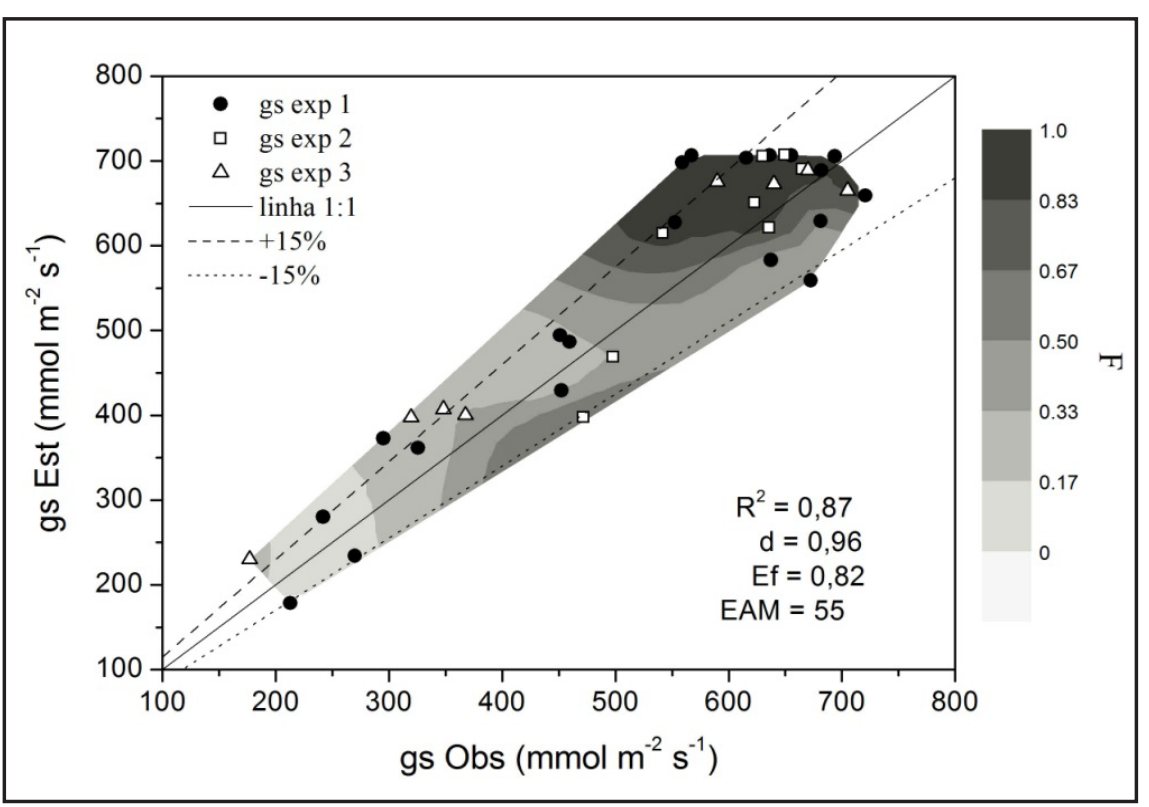

Figura 3. Comparação das condutâncias estomáticas ( $g s)$ observadas ( $g s$ Obs) e estimadas ( $g s$ Est). As áreas sombreadas de $\mathrm{F}$ representam exclusivamente o teor de água disponível do solo no momento em que as medições de gs foram realizadas \{comparison of stomatal conductance $(g s)$ between simulated ( $g s$ Est) and observed ( $g s$ Obs). The shaded areas of F represent the soil available water content at the moment $g s$ measurements were carried out\}. Belem, UFRA, 2012-2014.

vegetação desmontaram que plantas de feijão-caupi sob estresse hídrico, elevaram rapidamente e significativamente os níveis de prolína livre nas folhas (Amira \& Abdul, 2013). A prolína é um importante aminoácido osmorregulador que se liga às moléculas de água com a finalidade de manter o nível de hidratação da folha, protegendo membranas e outras estruturas celulares contra desidratação. Jones (2007) mencionou que o feijão-caupi apresenta característica isohídrica, ou seja, mantém o potencial hídrico foliar elevado, mesmo sob déficit hídrico severo, sendo um equívoco utilizar o potencial hídrico foliar, desta cultura, como indicador de estresse hídrico.

Observa-se na Figura 2 uma tendência brusca de redução da $g s$, para disponibilidade de água no solo inferior a $35 \%(\mathrm{~F}=0,35)$, sugerindo a existência de uma função, que penaliza significativamente a $g s$ a partir de um ponto crítico. Este resultado foi semelhante ao obtido por Cardona-Ayala et al. (2013), ao avaliarem a dinâmica da $g s$ de 14 genótipos de feijão-caupi (não incluindo o BR3-Tracuateua) em função do teor de água do solo, observaram uma função semelhante a obtida neste estudo, porém com um ponto crítico levemente superior ( $40 \%$ de água disponível), provavelmente em função das cultivares utilizadas e condições de tempo.

Apesar da interação de T, DPV e I ter uma ponderação complexa na resposta da $g s$, o produto das variáveis meteorológicas (prod) se mostrou uma ótima variável de predição. Carneiro et al. (2008) encontraram uma forte correlação $\left(\mathrm{R}^{2}=0,88\right)$ do prod com a resistência estomática $(r s=1 / g s)$ da cultura do eucalipto em condições irrigadas. Souza et al. (2011) incorporaram (1987) e obtiveram melhorias na simulação do rendimento da cultura da soja no estado do Pará.

A proposta de combinar o efeito do clima em uma única variável para estudos de simulação pode ser uma alternativa para os inúmeros ajustes e transformações matemáticas frequentemente requerida pelos modelos de gs, como por exemplo o modelo Jarvis (1976) e Ball et al. (1987), mesmo porque parâmetros de modelos empíricos não têm clara significância fisiológica, portanto a adição deles só aumentaria a complexidade de sua determinação.

A Figura 3 apresenta a comparação a referida relação ao MDC de Sinclair dos dados de gs observados em campo e estimados pelo modelo, com os seguintes parâmetros ajustados: $g s_{\max }=$ $755 ; g s_{i m}=46$; ini $=0,021$; fin $=0,026$; $\mathrm{A}=2 ; \mathrm{b}=5,91$ e $\mathrm{c}=0,009$, onde foi observado uma distribuição homogênea ao longo da linha $1: 1$, indicando que a eficiência do modelo não foi afetada nem pelos diferentes teores de água disponível no solo (F) e tampouco pelo produto das variáveis meteorológicas. De modo geral, o modelo explicou $87 \%$ $\left(\mathrm{R}^{2}=0,87\right)$ da variação observada com elevado grau de concordância $(d=96)$ e eficiência $(E f=0,82)$. $O$ erro absoluto médio (EAM) foi de $55 \mathrm{mmol} / \mathrm{m}^{2} / \mathrm{s}$, apresentando uma variação de $\pm 15 \%$ em $95 \%$ das observações e superestimativa média inferior a $30 \mathrm{mmol} / \mathrm{m}^{2} / \mathrm{s}$. Estes resultados foram semelhantes aos obtidos por modelos mais complexos de caráter semimecanístico, como o descrito por Jara-Rojas et al. (2009), o qual considera concentração de $\mathrm{CO}_{2}$ no mesofilo foliar, interceptação de luz e potencial hídrico foliar.

Percebe-se que o modelo não possui um padrão sistemático de resposta ao nível de água no solo, com ocorrência de superestimavas de $g s$ tanto para condições ótimas como para condições de menor disponibilidade de água no solo. Da mesma forma, foi observado subestimativas em distintas condições de umidade do solo. Esta característica demonstra a robustez do modelo, apesar de sua natureza semi-empírica, uma vez que não existe uma tendência sistemática de resposta em função da menor ou maior oferta de água; pelo contrário, é função também das variáveis meteorológicas observadas. Erros sistemáticos são normalmente encontrados em simulações, como no trabalho de Souza et al. (2011) onde foram identificadas superestimativas e subestimativas da produtividade da soja sob condições de tempo seco e úmido, respectivamente. Segundo os autores, tais respostas ocorreram devido penalizações por alagamento aplicadas no balanço de nitrogênio, mas que na prática real são eliminadas quando são realizadas adubações concomitantemente com a fixação biológica, processo que infelizmente não é considerado pelo modelo.

A inclusão do efeito da água no solo 
na simulação da $g s$ tem sido utilizada por alguns pesquisadores como uma forma de garantir uma resposta aproximada do real papel dos estômatos no controle fisiológico que ocorre principalmente em condições de estresse hídrico, uma vez que as demais variáveis climáticas (T, DPV, I $\mathrm{I}_{\mathrm{o}}$ possuem maior importância no controle do processo físico da evaporação, ao contrário dos processos fisiológicos (Ortega-Farias et al., 2004).

Ortega-Farias et al. (2004) analisaram o efeito combinado das condições atmosféricas e de umidade do solo na estimativa da $r s$ do dossel e incorporaram no modelo de Penman-Monteith obtendo simulações do fluxo de calor latente com erros menores que $6 \%$, resultado bastante significativo para o cálculo da evapotranspiração da cultura.

O modelo de evapotranspiração de Penman-Monteith baseado no balanço de energia possui um fator de resistência à difusão de vapor d'água, da copa para atmosfera, chamado de resistência de superfície o qual representa uma regulação fisiológica da cultura. Portanto a determinação da resistência estomática $(r s=1 / g s)$ e sua recíproca, condutância, constitui-se um tópico chave na simulação da evapotranspiração (Vico et al., 2013). O bom desempenho do modelo sugere que seu mecanismo pode ser aplicado diretamente no cálculo da evapotranspiração real da cultura para avaliar o efeito do estresse hídrico no rendimento, uso eficiente da água, práticas de manejo e até do impacto das mudanças climáticas na demanda hídrica da cultura (Souza et al., 2011), uma vez que na região nordeste no estado no Pará o principal fator de risco climático se deve à sazonalidade da precipitação.

O modelo proposto neste estudo foi sensível aos teores de água no solo $(\mathrm{F}) \mathrm{e}$ as variações combinadas de $\mathrm{T}$, DPV e $\mathrm{I}_{0}$. A estrutura simples do modelo em questão não pode explicar o funcionamento dos estômatos independentemente do limbo foliar, porém a boa eficiência em predizer a resposta da $g s$, em folhas intactas, sugere que as expressões matemáticas utilizadas devem ser análogas às que descrevem o funcionamento real. Portanto, o mecanismo pode ser utilizado em futuros estudos de modelagem agrometeorológica na região de realização deste experimento, com potencial de expansão, uma vez que a temperatura do ar, umidade relativa do ar e irradiância solar possuem um padrão semelhante de variabilidade em grande parte da região amazônica.

\section{AGRADECIMENTOS}

Os autores agradecem ao $\mathrm{CNPq}$ pelo financiamento da pesquisa através do projeto Universal (processo $\mathrm{n}^{\circ}$ 483402/2012-5). O primeiro autor também agradece à CAPES pela concessão da bolsa de mestrado.

\section{REFERENCIAS}

AMIRA, MS; ABDUL, Q. 2013. Osmotic adjustment and yield of cowpea in response to drought stress and chitosan. Indian Journal of Applicad Research 13: 3-10.

ARISTIDES, F; LIMA FILHO, A; COELHO FILHO, MA; HEINEMANN, AB. 2013. Determinação da época de semeadura do feijão caupi no Recôncavo Baiano através do modelo CROPGRO. Revista Brasileira de Engenharia Agrícola e Ambiental 17: 1294-1300.

BALL, JT; WOODROW, IE; BERRY, JA. 1987. Progress in photosynthesis research. In: BIGGENS J (ed). A model predicting stomatal conductance and its contribution to the control of photosynthesis under different environmental conditions. The Netherlands: Martinus Nijhoff Publishers. p.221-224.

BARTERNG, P; HOOGENBOOM, G; PATANOTHAI, A; SINGH, P; WANI, SP; PATHAK, P; TONGPOONPOL, S; ATICHART, S; SRIHABAN, P; BURANAVIRIYKUL, S; JINTRAWET, A; NGUYEN, TC. 2010. Application of the cropping system model (CSM)-CROPGRO-Soybean for determining optimum management strategies for soybean in tropical environments. Journal Agronomy and Crop Science 196: 231-242.

BASTOS, EA; FERREIRA, VM; SILVA, CB; ANDRADA JUNIOR, AS. 2008. Evapotranspiração e coeficiente de cultivo do feijão-caupi no Vale do Gurguéia, Piauí. Irriga 13: 82-90.

CARDONA-AYALA, CE; JARMA-OROZCO, A; HARAMÉNDIZ-TATIS, A; PERNETHMONTAÑO, M; VERGARA-CÓRDOBA, CA. 2013. Gas Exchange and mass distribution of the cowpea (Vigna unguiculata (L.) Walp.) under water deficit. Agronomía Colombiana. 31: 288-296.

CARNEIRO, RLC; RIBEIRO, A; HUAMAN, CAM; LEITE, FP; SEDIYAMA, GC; CÉSAR NEVES, JCL. 2008. Consumo de água em plantios de eucalipto: Parte 2: modelagem da resistência estomática e estimativa da transpiração em tratamentos irrigados e nãoirrigados. Revista Árvore 32: 11-18.
CARVALHO, JJ; SAAD, JCC; BASTOS, AVS; NAVES, SS; SOARES, FAL; VIDAL, VM. 2014. Teor e acúmulo de nutrientes em grãos de feijão comum em semeadura direta, sob déficit hídrico. Irriga, Edição Especial 01: 104-117.

DAMOUR, G; SIMONNEAU, T; COCHARD, H; URBAN, L. 2010. An overview of models of stomatal conductance at the leaf level. Plant, Cell and Environment 33: 1419-1438.

GAO, Q; ZHAO, P; ZENG, X; CAI, X; SHEN, W. 2002. A model of stomatal conductance to quantify the relationship between leaf transpiration, microclimate and soil water stress. Plant, Cell and Environment 25: 1373-1381.

JARA-ROJAS, F; ORTEGA-FARÍAS, S; GÓMEZ, HV; POBLETE, C; POZO, AD. 2009. Model validation for estimating the leaf stomatal conductance in cv. Cabernet Sauvignon Grapevines. Chilean Journal of Agricultural Research 69: 88-96.

JARVIS, PG. 1976. Interpretation of variations in leaf water potential and stomatal conductance found in canopies in field. Philosophical Transactions of the Royal Society of London Series B-Biological Sciences 273: 593-610.

JONES, HG. 2007. Monitoring plant and soil water status: established and novel methods revisited and their relevance to studies of drought tolerance. Journal of Experimental Botany 58: 119-130.

LIMA, JRS; ANTONIO, ACD; SOARES, WA; SILVA, IF. 2006 Estimativa da evapotranspiração do feijão caupi utilizando o modelo de Penman-Monteith. Irriga 11: 477-491.

LOBET, G; PAGÈS, L; DRAYE, X. 2014. A modeling approach to determine the importance of dynamic regulation of plant hydraulic conductivities on the water uptake. Dynamics in the Soil-Plant-Atmosphere System 290: 65-75.

MONTEITH, JL. 1995. A reinterpretation of stomatal responses to humidity. Plant Cell Environ 18: 357-364.

MORADI, R; KOOCHEKI, A; MAHALLATI, MN; MANSOORI, H. 2013. Adaptation strategies for maize cultivation under climate change in Iran: irrigation and planting date management. Mitigation and Adaptation Strategies for Global Change 18: 265-284.

ORTEGA-FARIAS, S; OLIOSO, A; ANTONIOLETTI, R. 2004. Evaluation of the Penman-Monteith model for estimating soybean evapotranspiration. Irrigation Science 23: 1-9.

PAIVA, AS; FERNANDES, EJ; RODRIGUES, TJD; TURCO, JEP. 2005. Condutância estomática em folhas de feijoeiro submetido a diferentes regimes de irrigação. Engenharia Agrícola 25: 161-169.

PIERUSCHKA, R; HUBER, G; BERRY, JA. 2010. Control of transpiration by radiation. Proceedings of the National Academy of Sciences of the United States of America 107: 13372-13377.

REBELlO, FK; COSTA, AJG; FIGUEIRÓ, CLM. 2011. Conjuntura da produção e comercialização do feijão-caupi no Nordeste 
Paraense: safra 2010. Contexto Amazônico. Disponível em: http://www.bancoamazonia. com.br/images/arquivos/institucional/ biblioteca/boletim/contexto_amazonico_4. pdf. Acessado em 09 setembro 2015.

RODRIGUES, HJB; COSTA, RF; RIBEIRO, JBM; FILHO, JDCS; RUIVO, MLP; JÚNIOR, JAS. 2011. Variabilidade sazonal da condutância estomática em um ecossistema de manguezal amazônico e suas relações com variáveis meteorológicas. Revista Brasileira de Meteorologia 26: 189-196.
SILVA, CDS; SANTOS, PAA; LIRA, JMS SANTANA, MC; JÚNIOR, CDS. 2010. Curso diário das trocas gasosas em plantas de feijãocaupi submetidas a deficiência hídrica. Revista Caatinga 23: 7-13.

SINCLAIR, TR. 1987. Water and nitrogen limitations in soybean grain production. I. Model development. Field Crops Research 15: 125-141.

SOUZA, PJOP; RIBEIRO, A; ROCHA, EJP; BOTELHO, MN; SOUSA, AML; SOUZA, EB; FARIAS, JRB. 2011. Impacts of soyabean expansion on the Amazon energy balance: a case study. Experimental Agriculture 47: 553-567.

TACK, JB; UBILAVA, D. 2015. Climate and agricultural risk: measuring the effect of ENSO on U.S. crop insurance. Agricultural Economics 46: 245-257.

VICO, G; MANZONI, S; PALMROTH, S; WEIH, M; KATUL, GA. 2013. Perspective on optimal leaf stomatal conductance under $\mathrm{CO}_{2}$ and light co-limitations. Agricultural and Forest Meteorology 41: 56-65. 